Revue

de Sémantique

et Pragmatique
Revue de Sémantique et Pragmatique

39 | 2016

Varia

Intensité, anaphoricité et attitude énonciative : le cas de décidément

Intensity, anaphoricity and enunciative attitude: the case of décidément

\title{
Mongi Kahloul
}

\section{(2) OpenEdition}

\section{Journals}

Édition électronique

URL : http://journals.openedition.org/rsp/418

DOI : $10.4000 /$ rsp. 418

ISSN : 2610-4377

Éditeur

Presses universitaires d'Orléans

Édition imprimée

Date de publication : 1 octobre 2016

Pagination : 59-73

ISSN : 1285-4093

\section{Référence électronique}

Mongi Kahloul, «Intensité, anaphoricité et attitude énonciative : le cas de décidément », Revue de Sémantique et Pragmatique [En ligne], 39 | 2016, mis en ligne le 01 octobre 2017, consulté le 01 mai 2019. URL : http://journals.openedition.org/rsp/418 ; DOI : 10.4000/rsp.418 


\section{INTENSITÉ, ANAPHORICITÉ ET ATTITUDE ÉNONCIATIVE : LE CAS DE DÉCIDÉMENT}

Mongi Kahloul

ISLG / ICAR (CNRS UMR 5191 - Lyon 2 / ENS)

\section{INTRODUCTION}

L'adverbe décidément comme adverbe d'énonciation a été décrit comme un marqueur de «classification dissimulée» (Ducrot et al. 1980), du fait que, qu'il soit employé seul, avant ou après une phrase, il se présente en effet comme un jugement porté sur un ensemble de faits antérieurs à celui qui motive son expression (que véhicule la première phrase dans les échanges suivants) :

- Mathilde ne viendra pas ce soir - Décidément!

- Mathilde ne viendra pas ce soir-Décidément, je n'ai pas de chance avec elle!

Ces faits sont rassemblés sur la base d'un point commun qui reste implicite : en l'occurrence, la réplique, dans les deux enchaînements, manifeste, de la part du locuteur, un certain sentiment négatif (lassitude, découragement, tristesse, agacement...) face à ce qu'il apprend, présenté par décidément (dorénavant $D C D$ ) comme le dernier avatar d'une longue liste d'événements, ou comportements (on le verra plus loin) similaires, sans que l'on sache en quoi consiste cette similarité.

Le but ici n'étant pas de reprendre la description de Ducrot et al (1980), mais celui de prendre appui sur certaines remarques intéressantes fournies par les auteurs qui serviront de point de départ pour notre hypothèse dans l'analyse de l'adverbe décidément en tant qu'adverbial discursif. Cette étude trouve sa place dans les fonctions discursives des adverbiaux et propose de partir d'une catégorie dite adverbe en -ment qui a donné lieu à de nombreux travaux, en particulier classificatoires, faisant désormais partie des acquis de la linguistique. 
Ces travaux constituent des références obligées dans le domaine : Greenbaum 1969, Huang 1975, Mørdrup 1976, Schlyter 1977, Mélis 1983, Molinier 1990, Guimier 1996, Molinier et Lévrier 2000 et plus récemment Molinier 2009, Anscombre 2009 et bien d'autres encore décrivant la modalité de l'attitude énonciative.

Il est vrai, en prenant la parole, le locuteur ne se limite pas à «dire» un énoncé $\mathrm{E}$, mais peut exprimer «en disant» une attitude vis-à-vis de sa production énonciative. En nous appuyant sur le cadre théorique de l'énonciation en général, notre objectif à travers cette étude, est de procéder à l'analyse de l'emploi discursif de l'adverbe décidément. Notre hypothèse de départ consiste à voir dans l'usage sémantico-pragmatique - dans la façon d'opérer de cet item - le dessein de deux caractéristiques : l'anaphoricité d'une part, et celle de véhiculer une attitude énonciative à polarité négative d'autre part.

Avant de commencer à proprement parler l'analyse, nous voudrions préciser qu'il ne sera question ici que de l'adverbe placé en tête de l'énoncé, suivi ou non d'un co-texte droit, et non des autres emplois, notamment intraphrastiques.

\section{DU POINT DE VUE SYNTAXIQUE}

$D C D$, dans notre perspective, n'admet que la position frontale de l'énoncé (il thématise le «cadre du discours» (Anscombre 1989)).Cela n'exclut pas son apparition au milieu de l'énoncé : (Je jugeai qu'il valait décidément mieux partir plutôt qu'être chassé plus tard (Frantext)).

Ensuite, $D C D$ ne peut apparaître dans une clivée (1a), ni être interrogé (1b), ni nié $(1 \mathrm{c})^{2}$ :

(1) $D C D$, tout fout le camp! (Google, Anne Sinclair)

(a) *C'est $D C D$ que tout fout le camp.

(b) *Est-ce que $D C D$ tout fout le camp ?

(c) *Tout ne $D C D$ pas fout le camp.

${ }^{1}$ La dénomination la plus partagée ici est celle d'un adverbe d'énonciation à l'instar de honnêtement, franchement, sincèrement, etc. Guimier (1996: 6) parle d'adverbes exophrastiques qui sont externes à la phrase, en ce sens qu'ils ne participent pas à la construction de son sens référentiel, mais représentent des traces de l'intervention du locuteur, qui commente tout ou partie de son énoncé ou de l'acte qui le produit.

2 Anscombre (2009: 51) dans sa description du marqueur apparemment confirme ces propriétés : « (...) le apparemment 3 possède toutes les propriétés des adverbes dits d'énonciation : possibilité de la position frontale, impossibilité d'être ni nié ni interrogé, ou d'apparaître dans une clivée. Il est donc tout à fait comparable à un adverbe comme sincèrement dans ce même rôle d'énonciation. L'attitude énonciative que véhicule ce dernier est tout à fait claire. En disant par exemple Sincèrement, ta cravate te va mal, je parle depuis ma sincérité, je me présente comme sincère. Je ne décris pas mon énonciation comme étant sincère, je joue la sincérité. C'est en ce sens que les adverbes d'énonciation sont proches de certaines interjections. Ouf! exprime ainsi un soulagement que Je suis soulagé ne fait que décrire. » 


\section{DU POINT DE VUE SÉMANTIQUE}

D'emblée, nous pouvons remarquer que l'adverbe $D C D$, en soi, est la plupart du temps associé à une marque de subjectivité particulière :

(2) $D C D$, la discrétion n'était pas son fort ! (Frantext)

Nous avons ici une manifestation d'agacement et d'irritation véhiculée par le locuteur, restituée sur le mode présuppositionnel, face au comportement indiscret, répétitif selon le contexte, de la personne dont parle le locuteur.

Ensuite, afin de cerner quelque peu le sémantisme de l'adverbe, nous proposons une série de tests qui mettrait en exergue ce caractère exophrastique dont parlait Guimier.

\section{Test $1: D C D$ et les temps verbaux :}

(3) $D C D$, ce type ne comprend rien de rien !

(a) $D C D$, ce type n'a jamais rien compris !

(b) $D C D$, ce type ne comprendra jamais rien !

(c) $D C D$, ce type ne comprenait rien de rien!

(d) $D C D$, ce type ne comprit rien de rien.

Il est à noter que le changement du temps n'affecte en rien le sens véhiculé par l'adverbial $D C D$ qui est celui d'exprimer une certaine exaspération face à la répétition du caractère idiot de la personne en question. Il est évident que l'insertion de l'adverbe n'altère pas le sens référentiel de l'énoncé mais apporte un quelconque commentaire, traduisible selon notre hypothèse par «une attitude énonciative».

\section{Test $2: D C D$ et l'aspect :}

Toutes ces occurrences sont tirées de la base de données Frantext :

(4) $D C D$, le temps n'est pas encore venu pour vivre en paix ! (imperfectif)

(5) $D C D$, ça me poursuivra toute ma vie ! (duratif)

(6) $D C D$, j'allais de surprise en surprise ! (itératif)

(7) $D C D$, je deviens plus dur avec les gens. (inchoatif)

De même, la notion d'aspect ne présente aucun changement quant au sémantisme de $D C D$. En effet, ni la borne du procès pour vivre en paix qui n'est pas encore atteinte dans (4), ni le procès saisi dans sa durée visible à travers le verbe poursuivre dans (5), ni la succession des procès exprimés par de surprise en surprise saisis dans leur répétition dans (6), ni la saisie du procès à son début dénoté dans le verbe devenir, ne modifient le sens de l'adverbe $D C D$. Celuici porte déjà en lui (i.e. dans son noyau sémique spécifique) le sentiment de l'exaspération, même - comme on le verra plus loin - quand il est utilisé seul en tant qu'interjectif. Il est à noter que le point commun à ces énoncés est qu'ils dénotent tous un sens à «teneur dépréciative» : le constat de l'impossibilité de vivre en paix dans (4), un événement triste ou désagréable poursuivra le locu- 
teur durant toute sa vie dans (5), une note négative émane, selon le contexte, des surprises vécues par le locuteur dans (6), et enfin souligner un caractère nouveau chez le locuteur, en l'occurrence celui de devenir plus dur avec les gens.

Nous stipulons, comme autre propriété sémantique, le caractère potentiellement anaphorique de $D C D$. De ce fait, l'adverbe peut être dit «anaphorique», en ce que le commentaire qu'il exprime porte sur l'expérience passée en même temps que sur ce qui vient d'être appris ou constaté, qui constitue une sorte de «comble» : pour revenir sur les exemples supra, la non-venue de Mathilde apparaît comme la goutte qui a fait déborder le vase, comme ce qui couronne un tout du même acabit. Si, comme l'a montré l'article cité supra, décidément ne peut ainsi se confondre avec finalement (qui marque également une forme de clôture discursive), il ne peut pas non plus recevoir comme synonyme franchement, ainsi que le confirment les dictionnaires : ce dernier adverbe, en effet, n'est pas rétrointerprétatif (i.e. revenant mentalement sur des moments passés), et n'inclut aucunement le point de vue porté sur des procès répétitifs, dont la répétition génère le sentiment que véhicule ce point de vue : pour s'en convaincre examinons ces deux énoncés :

- Franchement, tout fout le camp!

- $D C D$, tout fout le camp !

L'autre propriété de l'adverbe franchement est qu'il n'est pas non plus axiologique, en ceci qu'il ne porte pas de jugement sur ce que rapporte l'énoncé - même si ce dernier peut asserter un énoncé négatif (Franchement, je suis déçu par le directeur !) - alors que $D C D$ témoigne, de par son emploi même, d'une certaine lassitude ou de quelque exaspération. Ainsi, l'énoncé comportant franchement se présente comme le constat, assumé par le locuteur (qui revendique la franchise de son propos) que «tout fout le camp», tandis que l'énoncé comportant $D C D$ ajoute à ce constat l'idée que ce dernier a été précédemment opéré un certain nombre de fois.

Par ailleurs, nous tenterons, toujours dans la perspective retenue, de montrer que $D C D$ ne participe pas au sens de l'énoncé en ceci qu'il est rétrointerprétatif mais qu'il serve à montrer (et non à décrire) au sens d'Anscombre (2009), une attitude se rapportant précisément à ces moments vécus ou à ces procès répétitifs.

\section{Test 3 : $D C D$ : marqueur anaphorique ?}

(8) $D C D$, il y a quelque chose entre moi et le public. Il faudra que j’y pense. (Frantext)

(a) *Avec décision, Il y a quelque chose entre moi et le public. Il faudra que j'y pense.

(b) *De façon décidée, il y a quelque chose entre moi et le public. Il faudra que j'y pense. 
(c) $* D C D$ parlant, il y a quelque chose entre moi et le public. Il faudra que j'y pense.

(d) $* A D C D$ parler, il y a quelque chose entre moi et le public. Il faudra que j'y pense.

(9) Franchement, ta cravate te va mal. (Anscombre)

(a) En toute franchise, ta cravate te va mal.

(b) Franchement parlant, ta cravate te va mal.

(c) À franchement parler, ta cravate te va mal.

Il ressort de ce test que l'emploi de Avec décision dans (8a) et De façon décidée dans (8b) exprime littéralement la ferme résolution par rapport à la réalisation du procès de l'énoncé et non la récurrence d'un procès vécu ou vu par le passé, d'où l'astérisque. Cet usage existe dans un des emplois de $D C D$ suggérant cette ferme résolution à mettre à tout prix en exécution un procès (10) :

(10) Ces politiciens qui font $D C D$ / de façon décidée / avec décision / franchement/ tout pour rester au pouvoir.

De plus, les dérivés de $D C D$ dans (10) expriment plutôt la volonté et l'acharnement à vouloir réaliser le procès (rester au pouvoir), idée exprimée aussi par Franchement qui affiche, d'une manière décidée et sans équivoque, l'opinion du locuteur. Il s'agit ici d'un haut degré de modalité témoignant de l'implication du locuteur mais il n'est aucunement question d'une opération cognitive anaphorique.

Le $D C D$ qui nous occupe promeut donc sémantiquement l'idée de la récurrence d'un événement ou d'un fait qui se révèle, pour le moins irritant, pour le locuteur. Il suffit d'introduire $D C D$ pour rendre compte de deux choses :

(i) Un jugement donné, traduit ensuite sous forme d'attitude, suite à une accumulation de faits analogues ou hétérogènes vécus ou accumulés dans la mémoire, actualisant par le truchement de $D C D$ tous les $\mathrm{F}_{\mathrm{n}}$ d'une manière «compressée» au moment $t_{0}$ de l'énonciation.

(ii) Une teneur (attitude) négative se dégage de l'énoncé qui va de la simple irritation au rejet le plus total du procès ou du comportement dont il est question.

De fait, l'adverbe franchement, donné comme synonyme par les dictionnaires de référence, ne partage pas les mêmes propriétés retenues pour $D C D$. En effet, dans (9), l'adverbe franchement est dénué de toute dimension de récurrence précisant que la cravate a déjà fait l'objet de critique. D'ailleurs les dérivés de (9), à savoir (9a), (9b) et (9c) corroborent l'idée du caractère immédiat de la remarque, dénuée de toute référence anaphorique renvoyant à d'autres remarques faites préalablement à propos de cette cravate. Le locuteur parle «depuis sa franchise» en formulant un propos P. Anscombre (2009 : 3), dans sa description des adverbes sincèrement, franchement, honnêtement, etc., nous 
confirme cette valeur sémantique particulière : «(...) en disant Sincèrement, ça ne casse pas des briques, on ne se décrit ni ne se présente comme sincère, on parle depuis sa sincérité », l'auteur parle (ibid.) d'attitude énonciative : « elle reflète en effet, d'un point de vue intuitif, une attitude énonciative vis-à-vis de l'énonciation. Et cette attitude n'est pas décrite mais montrée ». L'auteur ajoute (ibid. : 73), en comparant sincèrement à $D C D$ que : «Sincèrement me campe comme parlant à partir de ma sincérité, comme «parlant sincère», Décidément me montre comme me résignant à une certaine fatalité ».

Pour rendre compte de l'incompatibilité de l'emploi de $D C D$ dans des contextes non-anaphoriques, examinons ce qui suit :

(11) $* D C D /$ Franchement, c'est la première fois qu'il découche !

(12) DCD/Franchement, ce n'est pas la première fois qu'il découche !

Ducrot (1980 : 133) évoquait déjà l'idée de «regroupement» pour signifier la répétition du procès :

«Le locuteur laisse entendre qu'il a des raisons de retenir la propriété $\mathrm{C}$ qui fait apparaître la série $F_{1} \ldots F_{n}$ comme une succession d'événements analogues, comme une répétition. »

(11) est inacceptable puisque, précisément, l'idée de la répétition y est absente. L'insertion de $D C D$ s'avère injustifiée et impertinente, contrairement à franchement qui lui, peut très bien s'y combiner pour les raisons évoquées supra, en insistant néanmoins, sur la singularité de l'événement. Du point de vue pragmatique franchement peut laisser transparaître une attitude de la part du locuteur visant à défendre ou excuser la personne qui découche. Dans (12), la répétition du même procès rend légitime, sémantiquement, l'introduction de $D C D$ et même de Franchement qui peut très bien ici s'y combiner véhiculant une certaine lassitude ressentie par le locuteur, face à ce comportement de découcher qui se renouvelle.

Nous le savons, le caractère répétitif est à la base de cette attitude énonciative négative. Il est communément partagé que la notion de l'excès ou du «ras-le-bol» n'est pas ressentie au bout d'une seule occurrence, mais suite à la succession d'événements ou de comportements qualifiés de fautifs. Pour rendre compte de cette condition, nous comparons deux marqueurs partageant cette caractéristique.

Ducrot (1980 : 95) évoquait l'existence d'un «Mais de renforcement» qui se recoupe dans l'idée de la récurrence avec le marqueur qui nous occupe. En témoignent les phrases de notre corpus :

(13) Mais bois ta tisane, elle va refroidir !

(14) $D C D$, tu vas la boire cette tisane, elle va refroidir!

Il est vrai que l'anaphoricité ici est rendue possible au moyen de faits $\left(F_{n}\right)$ 
analogues dans les deux procès. De fait, on ne peut pas, en déposant la tisane devant l'invité, s'exclamer *Mais bois ta tisane !, deux conditions sont nécessaires pour légitimer l'emploi de mais ou de $D C D$ :

a) Remarquer à plusieurs reprises que l'invité ne touche pas à sa tisane. (phénomène mémoriel)

b) Le locuteur a déjà demandé à l'invité de boire la tisane en question (Fn). (la récurrence). (phénomène anaphorique)

Le corpus retenu nous offre un autre moyen où l'anaphoricité est véhiculée lexicalement :

(15) $D C D$, il n'en rate pas une !

(16) $D C D$, tu ne peux jamais arriver à l'heure !

(17) $D C D$, cette manie d'abandonner ses amis était sa perversion à elle !

(18) $D C D$, il s'agit bien d'un tueur en série.

(19) $D C D$, ça devient une habitude chez lui de découcher.

Tous ces énoncés renferment en eux l'idée de la «récurrence» et l'anaphoricité. Dans (15), l'expression figée n'en rate pas une présuppose que la personne dont parle le locuteur est à l'affut de tout ce qui se passe, dans (16) l'adverbe jamais présuppose que l'interlocuteur est un retardataire récidiviste, dans (17) le substantif «manie» laisse également entrevoir ce procès qui revient en boucle, confirmé par la définition qu'offrent les dictionnaires «Habitude bizarre et tyrannique, souvent agaçante ou ridicule». Dans (18), l'expression «en série» dénote la succession de meurtres perpétrés sur des victimes, avec le supplément d'information qui caractérise le rituel relatif à l'acte. Enfin, nous avons le même phénomène dans (19) : le substantif «habitude» confère à l'interprétation ce caractère répétitif aussi.

\section{DCD : UN MARQUEUR D'ATTITUDE ÉNONCIATIVE NÉGATIVE ?}

À la lumière de ce qui précède, on pourrait désormais formuler notre hypothèse. En effet, nous stipulons que $D C D$ obéit au schéma global d'un marqueur d'attitude énonciative dépréciative. Nous soutenons que l'adverbe en soi soustend l'idée de la «récurrence» d'une part, et véhicule des émotions comme l'irritation, l'exaspération, l'ironie et l'indignation, d'autre part.

À parcourir le corpus prévu à cette étude (Frantext, Google et des occurrences authentiques), plus que mille occurrences en tout, nous n'avons pas rencontré une seule véhiculant un constat positif par le truchement de $D C D^{3}$, ce qui

\footnotetext{
${ }^{3}$ Nous avons exposé le corpus à de francophones natifs et ont confirmé ce statut dépréciatif : selon eux même un énoncé d'apparence positif : «Décidément, il réussit chaque année à ses examens», peut laisser transparaître en filigrane une certaine jalousie ou un quelconque froissement de la part du locuteur, à voir la personne réussir à chaque fois.
} 
nous pousse à chercher la cause de cette insuffisance. Examinons ces énoncés :

(20) $* D C D$, il est toujours sur son trente et un! (antiphrase)

(21) * $D C D$, je suis doué pour les travaux manuels! (antiphrase)

(a) $D C D$, je ne suis pas doué pour les travaux manuels !

(22) $* D C D$, elle est très discrète! (antiphrase)

(a) $D C D$, la discrétion n'était pas son fort !

(23) $* D C D$, c'est un vrai cordon bleu! (antiphrase)

(a) $D C D$, elle cuisine toujours aussi mal !

Le corpus est fabriqué par nos soins, dans lequel nous avons tenté de combiner $D C D$ avec un procès positif (mélioratif) dans (20), (21), (22) et (23) pour tester la valeur sémantico-pragmatique du marqueur. L'idée est ici de cerner la provenance de cette «teneur négative». Force est de constater que la tentative se révèle concluante. En effet, être sur son trente et un dans (20), être doué pour les travaux manuels dans (21), être très discrète dans (22) et être un vrai cordon bleu dans (23) combinés à $D C D$ nous donnent des énoncés très peu naturels avec une gêne dans l'interprétation. De fait, la gêne (confirmée par des locuteurs natifs) provient probablement de l'incompatibilité entre les lexèmes marqués positivement et le fait qu'ils soient introduits par $D C D$. Toutefois, tous les énoncés sont parfaitement acceptables si l'on privilégie la piste antiphrastique : si (20) est dite pour décrire quelqu'un qui est toujours mal vêtu, alors l'usage de $D C D$ peut très bien fonctionner. De même, si le locuteur dans (21) voulait mettre l'accent sur le contre-pied de la vérité assertée, à savoir qu'il n'est pas doué pour tout ce qui est manuel, alors le marqueur pourrait être acceptable ici. En définitive, la série dotée de (a) dans (21a), (22a) et (23a), est fournie pour restituer ce caractère négatif et serait l'explicitation de ce qui est implicité au moyen de l'antiphrase grâce à la négation syntaxique ne...pas, ou lexicale (mal).

Afin de nous assurer du fait que la propriété de la teneur «dépréciative» est bel et bien inhérente au signifié même du marqueur $D C D$, nous procédons à la commutation par Franchement :

(20b) Franchement, il est toujours sur son trente et un.

(21b) Franchement, je suis doué pour les travaux manuels.

(22b) Franchement, elle était très discrète.

(23b) Franchement, c'est un vrai cordon bleu!

La série (20b) jusqu'à (23b) introduite au moyen de l'adverbe franchement pour lequel les dictionnaires nous offrent les définitions suivantes «sans hésitation, d'une manière décidée : y aller franchement», mais aussi «sans équivoque, nettement, poser franchement un problème», présente un haut degré de subjectivité énonciative fournie sur le mode explicite, réduisant les énoncés à de simples assertions sans aucune attitude énonciative. 
Un dernier test, consistant en l'omission de $D C D$ dans les énoncés ci-dessous, pourrait cerner l'apport sémantico-pragmatique du marqueur quant à cette teneur «dépréciative» :

$\varnothing$, il est toujours sur son trente et un.

$\varnothing$, je suis doué pour les travaux manuels.

$\varnothing$, je ne suis pas doué pour les travaux manuels.

$\emptyset$ elle était très discrète.

$\emptyset$, la discrétion n'était pas son fort.

$\varnothing$, c'est un vrai cordon bleu.

$\varnothing$, elle cuisine toujours aussi mal.

Nous confirmons donc que l'omission du marqueur $D C D$ lève totalement l'ambiguïté en dissipant la double interprétation de l'attitude énonciative et confère aux énoncés une neutralité dans l'interprétation en les réduisant à de simples assertions.

\section{DCD ET L'ANAPHORE MÉMORIELLE}

À y voir de plus près, nous avons pu relever des occurrences dans notre corpus où le processus de l'anaphoricité ne s'opère pas forcément par le phénomène de la «récurrence» ou de la répétition, mais que la coréférentialité emprunte un autre parcours, cette fois-ci relatif à la cognition. À ce propos, les cognitivistes évoquent l'importance de l'élément appelé «le savoir partagé» où la source de ce savoir peut être déclenchée dans la mémoire du locuteur par un mécanisme mettant en marche le vécu ou l'expérience des moments désagréables, tels les aléas de la vie, l'acharnement du sort, l'animosité du milieu social ou professionnel. En effet, c'est une opération mentale consistant à replonger par la mémoire dans le passé, proche ou lointain, en actualisant par la pensée, ces mauvais événements. Nous pouvons gloser cela par : «Avec du recul, vu tous les X que j'ai pu voir ou vivre ou partager, je ne peux qu'admettre au bout du compte, le caractère $\mathrm{C}$ « dépréciatif » dû à cette succession de $\mathrm{X}^{\mathrm{n}}$.

Ducrot (1980 : 133) évoquait déjà l'idée de «regroupement» qu'il définissait comme suit : «(...) En parlant de regroupement, nous voulons dire que les faits $F_{1} \ldots F_{n}$ sont présentés comme analogues, comme partageant une caractéristique $\mathrm{C}$. »

Cette idée de faits analogues est reprise dans cet autre passage : «De plus, en employant décidément !, le locuteur laisse entendre qu'il a des raisons de retenir la propriété $C$ qui fait apparaître la série $F_{1} \ldots F_{n}$ comme une succession d'événements analogues comme une répétition. » (op. cit.)

La condition pour légitimer l'emploi de $D C D$, selon Ducrot, est que les faits $\left(\mathrm{F}_{\mathrm{n}}\right)$ doivent être identiques ( $c f$ : analogues et répétition). Pour expliciter 
cette succession, il illustrait par les exemples de «la machine à laver qui n'arrête pas de tomber en panne», «les collisions des barques» ou encore «les pannes de l'ascenseur».

Si l'idée toute ducrotienne de la «succession» $\left(\mathrm{F}_{\mathrm{n}}\right)$ s'avère intéressante et tout à fait pertinente dans l'emploi de $D C D$, nous pouvons tout de même la nuancer, en posant, l'hypothèse que les $\left(\mathrm{F}_{\mathrm{n}}\right)$ puissent être de nature diverses et qu'il ne s'agit pas toujours du même fait qui se répète.

Les occurrences tirées du corpus tendent à le prouver :

(24) $D C D$ ! ' j'ai toujours considéré la guerre comme une immense connerie ! (authentique)

(25) $D C D$ ! je n'étais pas faite pour le bonheur ! (Frantext)

(26) $D C D$ ! on n'est pas sorti de l'auberge! (authentique)

(27) $D C D$ ! tout fout le camp ! (authentique)

Nous soutenons que l'anaphore mémorielle au sens de Kleiber (1994) est un parcours cognitif qui rend manifeste le référent et s'érige en un processus qui promeut le critère de la saillance de choses ou d'événements déjà connus ou vécus par le passé. L'actualisation de ces moments en $\mathrm{t}_{0}$ est soumise à l'évaluation du locuteur qui finit par exprimer son attitude.

Le schéma global qui retrace un peu ce parcours mémoriel serait :

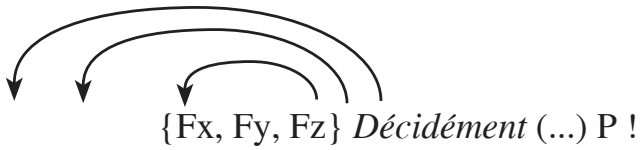

Afin de vérifier ce schéma, nous analysons ce corpus à la lumière de la définition offerte par Kleiber (1994) pour montrer ce flashback représenté ici par les flèches. En effet, dans (24) le locuteur choqué par l'image d'enfants syriens déchiquetés à la télévision, exprime avec beaucoup d'amertume et d'horreur son attitude à propos de la guerre.

Il est important de préciser ici, en plus du caractère complètement interjectif, quelques effets prosodiques et intonationnels accompagnant la prononciation de $D C D$ dans cette perspective. En effet, le locuteur prononce le marqueur en traînant sur les syllabes, d'une manière quasi syllabique (dé-ci-dé-ment), suivie d'une petite pause (...) comme pour se donner le temps de replonger (i.e. flashback) au moyen de la mémoire, dans les événements suscitant l'atrocité de la guerre, passant en revue les maux et les dégâts occasionnés déclenchés

${ }^{4}$ Le point d'exclamation offre à $D C D$ le statut interjectif à part entière. Le locuteur fait une très brève pause avant de donner l'énoncé postposé au marqueur $D C D$. 
par l'image qu'il vient de voir. Le locuteur peut ne pas être passé par l'expérience de la guerre, mais c'est plutôt l'idée qu'il se fait de la guerre, avec ses conséquences néfastes, qui refait surface dans sa mémoire. Nous le savons, les horreurs de la guerre sont de nature diverses : la destruction, la mort, la mutilation, la disparition, etc. C'est précisément ce que nous désignons par $\mathrm{F}_{\mathrm{x}}, \mathrm{F}_{\mathrm{y}}$, $\mathrm{F}_{\mathrm{Z}}$, etc, dans notre schéma. Il ne s'agit donc pas d'une répétition du même $\mathrm{F}$ mais de plusieurs $\left(\mathrm{F}_{\mathrm{n}}\right)$ hétérogènes, gravitant ici autour du champ lexical de la guerre. Du point de vue pragmatique, le marqueur $D C D$ introduit l'énonciation $(\mathrm{P})$ après une auto-évaluation et une délibération cognitive rétrospective, provoquant par là-même une émotion traduisant cette attitude négative, élucidée dans le lexème péjoratif (connerie) dans (24).

Le même schéma se reproduit dans (25) dans la mesure où le locuteur (une femme) fait le bilan de sa vie par un monologue intérieur, passant en revue tous les aléas qui ont fait obstruction à son bonheur en tant que femme. Il va sans dire que l'acharnement du sort contre elle est restituable anaphoriquement à travers $(\mathrm{P})$, suggérant la forte déception, doublée d'un sentiment de résignation devant le constat négatif en matière de bonheur. Il est à noter que les Fn ici sont également de natures diverses : la vie conjugale $\left(\mathrm{F}_{\mathrm{x}}\right)$, professionnelle $\left(\mathrm{F}_{\mathrm{y}}\right)$, familiale $\left(\mathrm{F}_{\mathrm{z}}\right)$, expériences personnelles $\left(\mathrm{F}_{\mathrm{n}}\right)$ décevantes ou malheureuses qui peuvent parfois remonter à l'enfance (maltraitance, viol, etc.).

L'énonciation de $D C D$ joue, en quelque sorte, le rôle de catalyseur servant à déclencher la réaction après un flashback qui retrace tout ce vécu ${ }^{5}$ qui a pu porter malheur au locuteur.

Dans (26), nous assistons au même phénomène de restitution mentale (i.e. la saillance). C'est un exemple authentique qui s'est passé lors d'une discussion sur la révolte tunisienne et les événements actuels qui ne cessent d'entraver la transition démocratique. Comme nous l'avons précisé supra, $D C D$ présuppose qu'il y a eu d'autres événements auparavant jugés négatifs (d'où l'anaphoricité) et qu'à chaque fois le locuteur est déçu par la tournure que prend la révolution. En effet, c'est le cumul des déceptions causées au locuteur par les revendications des ouvriers $\left(\mathrm{F}_{\mathrm{x}}\right)$, le barrage des routes $\left(\mathrm{F}_{\mathrm{y}}\right)$, les conflits entre les différents partis $\left(\mathrm{f}_{\mathrm{z}}\right)$, etc., qui crée ce sentiment d'incertitude et de malaise suite à ce bilan négatif, justifiant par là-même l'usage de $D C D$.

${ }^{5}$ Nous faisons référence ici à la notion de saillance évoquée par Kleiber, notion employée en sémantique pour décrire la centralité de certains référents dans la conscience du locuteur. Cette accessibilité référentielle est rendue possible par une saillance locale, dans la mesure où les événements sont encore présents dans la conscience du locuteur ; mais aussi par une saillance cognitive en ceci que les événements en question refont surface dans la mémoire du locuteur une fois déclenchés par $D C D$. 
L'énoncé (27) vient confirmer ce calcul cognitif, l'indice en est le pronom indéfini tout qui, selon le TLFi, exprime à la fois « la totalité d'une série » et «la plénitude d'une réalité ». En effet, ce pronom, considéré dans la perspective de l'attitude énonciative, explicite l'idée holonymique des événements envisagés par le locuteur, d'une manière 'compressée', suscitant la résignation ${ }^{6}$ et l'incapacité devant ce constat négatif. Du point de vue pragmatique, la rétrointerprétation des $\mathrm{F}_{\mathrm{n}}$ engagée par $D C D$ pourrait, toutes choses étant égales par ailleurs, être équivalente à d'autres locutions à l'instar de Tout bien pensé (TBP), Tout compte fait (TCF), Tout bien considéré (TBC) ou Tout bien pesé (TBP), placées également en position frontale. Deux différences sont, néanmoins, à noter : d'abord, ces locutions ne sont pas foncièrement d'attitude énonciative négative dans la mesure où ils peuvent introduire un constat positif (28), ce qui n'est pas le cas pour $D C D$ :

(28) $T B P / T C F / T B C / T B P / * D C D /(\ldots)$, ce temps de Lisbonne valait la peine d'être vécu. (Frantext)

L'explication qui s'impose à nos yeux, pour confirmer le caractère anaphorique de ces locutions, provient déjà de leur aspect perfectif, dû à la forme participiale, qui précise que le locuteur intervient après le terme de l'opération cognitive ${ }^{7}$. (Cf. le schéma Supra).

Ensuite, le sens de la récurrence et de l'anaphoricité n'est pas contenu dans le signifié même des locutions comme c'est le cas pour $D C D$. En témoigne l'usage interjectif, dans la structure monologique ${ }^{8}$, sans le (P), cotexte droit dans (29) :

(29) Décidément!/ *TBP !/ *TCF !/ *TBC!/ *TBP !

À l'oral, un locuteur, en replongeant dans son passé, proche ou lointain, pour faire un bilan quelconque, dont le constat négatif n'était aucunement pré-

${ }^{6}$ Anscombre (2009 : 73) dans la perspective énonciative dit ceci : « (...) tant franchement adverbe d'énonciation que tout 'renforçateur' sont des marqueurs d'attitude énonciative. En utilisant un adverbe d'énonciation, on montre l'attitude qui régit notre vision des événements. Sincèrement me campe comme parlant à partir de ma sincérité, comme 'parlant sincère', décidément me montre comme me résignant à une certaine fatalité ».

${ }^{7}$ Schelling (1982 : 156) en statuant sur le sémantisme des particules Compte vs Somme dans tout compte fait et en somme stipule que l'opération suggérée par le sémantisme de Compte est plus complexe que celle suggérée par le mécanisme de Somme, car avec l'expression faire «la somme de » les entités sont additionnées, c'est une procédure proche d'une opération mathématique ; tandis que l'expression «faire les comptes» exclut la possibilité de mentionner « ce qui a été compté »; il s'agit donc d'une procédure qui inclut d'autres opérations que celles d'ordre exclusivement mathématique et qui implique alors plus fortement le raisonnement du sujet.

${ }^{8}$ Nous opposons ici monologique à dialogique. Il arrive souvent que locuteur se transforme en un 'tu' dans la mesure où il est à la fois l'émetteur et le récepteur. 
visible ni même envisagé, sous le choc et l'étonnement, aggravés par une prise de conscience subite, peut dans une interjection employer $D C D$ !, mais il est impossible de le faire avec les locutions prises en considération dans (29), d'où l'astérisque.

Pour finir, le marqueur $D C D$ !, sous sa forme interjective, peut même être produit face à un comportement répétitif jugé irritant ou impoli d'un tiers. Prononcé avec une certaine intonation, et à voix basse, le marqueur à lui seul renferme à la fois la récurrence et l'acharnement, véhiculant cette attitude énonciative «dépréciative» du locuteur. En revanche, l'impossibilité des locutions dans (29) d'apparaître dans la structure interjective est certainement due à leur statut conclusif ici qui exige un cotexte droit à $(\mathrm{P})$, faute de quoi leur usage serait incohérent voire elliptique. De ce fait, faire l'économie ici de (P) laisserait l'interlocuteur insatisfait dans la mesure où il s'attend à un résultat après la délibération « cognitive » du locuteur, devant normalement déboucher sur un (P) conclusif ou reformulatif. De plus, le $D C D$ interjectif peut embrayer sur la situation d'énonciation, sur le mode d'intervention (monologal) et véhiculer un sentiment négatif. Rouanne (2010 : 53), en décrivant l'interjection, nous confirme cette idée :

«Ces interjections ne sont pas énoncées pour apporter une information, elles n'ont pas de contenu descriptif. Elles acquièrent leur valeur de et par l'énonciation : l'énonciateur laisse transparaître son étonnement, son admiration, sa contrariété, son indignation. »

En témoigne le contexte où un locuteur excédé par le comportement du voisin qui jette la poubelle dans la rue pour une énième fois, peut s'exclamer : Décidément!

Nous avons là un cas qui ne nécessite pas de cotexte droit explicitant l'emploi de $D C D$ car l'enchaînement se fait dans le cadre du discours, dans un « espace discursif » au sens d'Anscombre (1990) : le témoin oculaire a déjà vu ce comportement à plusieurs reprises par le passé et c'est une fois de plus. Il s'agit donc d'une attitude énonciative véhiculant le rejet de ce comportement, jugé inadmissible aux yeux du locuteur.

\section{CONCLUSION}

L'analyse monographique de l'adverbe $D C D$ nous a montré, grâce aux tests aussi bien syntaxiques que sémantiques que, en sa qualité de marqueur discursif, il est employé pour mettre en exergue une intensité dans l'expression sous-tendue par l'idée de 'l'acharnement', que celui-ci soit attribué au sort ${ }^{9}$ et

${ }^{9}$ Nous renvoyons ici à l'idée de «la force des choses» préconisée par Ducrot (Op.cit.) confirmant le caractère incontrôlable des événements, trouvant en eux un élément hostile rejoignant l'idée de fatalité. 
donc indépendamment du locuteur, ou alors attribué à une personne considérée comme actant effectif de cet acharnement, infligé sous plusieurs formes, au locuteur. A travers les tests nous avons pu montrer que ni le temps ni l'aspect n'ont d'incidence sur le sémantisme du marqueur. En effet, en ce qui concerne le mode de son instruction sémantique $D C D$ n'est tributaire ni du cotexte ni même du contexte interlocutif, comme le serait par exemple le connecteur D'ailleurs, qui exige un cotexte gauche justifiant son emploi. De plus, concernant l'anaphoricité du marqueur, nous pouvons affirmer, que là encore il n'a nul besoin de contexte linguistique particulier pour remonter à l'antécédent, source de cette anaphoricité, mais que cela se fait automatiquement d'une manière autoréférentielle ${ }^{10}$. Nous voulons dire par là que prononcer $D C D$ nous renvoie de façon systématique à d'autres événements situés dans le passé

Le test des dérivés de $D C D$ (Cf. (8a, 8b et 8c)) et la commutation par Franchement témoignent de l'éloignement de la perspective anaphorique. Nous avons poussé l'analyse - pour cerner la provenance de cette récurrence - en prouvant la non-compatibilité du marqueur avec un procès semelfactif (Cf. (11)). Quant à l'hypothèse de l'attitude énonciative négative, elle se trouve, nous le pensons, vérifiée grâce aux deux propriétés élucidées par le phénomène de récurrence d'une part, et par le fait que $D C D$ suggère des émotions négatives, telles : l'exaspération, l'irritation, l'indignation, d'autre part. Pour ce faire, nous avons pu démontrer l'incompatibilité du marqueur avec des énoncés positifs, (Cf. (2O), (21), (22) et (23)), et confirmer par là-même, grâce au test de l'omission, sa propriété sémantique spécifique, celle de se combiner qu'avec des constats jugés négatifs.

En outre, nous avons pu montrer que $D C D$ opère au moyen de la saillance cognitive dans la manière de passer en revue les faits, ou événements $(\mathrm{x}, \mathrm{y}, \mathrm{z} \ldots)$ (Cf. (24) à (27)). Pour finir, nous avons ouvert une autre piste considérant $D C D$ ! en tant qu'interjection à part entière, où il s'érige en tant que marqueur d'attitude énonciative négative. Un second indice qui conforte notre hypothèse de départ est que $D C D$ non seulement enregistre la rupture avec une situation qui finit par apparaître ingérable (haut degré) au sens d'Anscombre (2009), mais aussi montre cette attitude énonciative négative (de pessimisme) : "Décidément, Caen n'est pas fait pour vibrer en coupe !» (Titre d'un journal concernant une équipe de football). L'énoncé est ici l'écho d'un espoir constamment déçu (celui que l'équipe de Caen remporte une victoire), et l'attitude énonciative dépréciative est rendue possible grâce au truchement du marqueur $D C D$, qui véhicule la leçon à tirer une bonne fois de multiples observations, aggravant

${ }^{10}$ Par 'autoréférentielle' (faute de mieux), nous voulons dire «allant de soi», c'est-àdire que décidément porte en lui l'idée de la récurrence. 
le poids du jugement porté, glosable par «inutile de continuer à s'illusionner sur l'avenir de l'équipe de Caen». On cesse donc de s'interroger ou, a fortiori, d'espérer : le marqueur $D C D$ engage à tirer la leçon sans plus tergiverser et à adopter un autre point de vue sur l'équipe en question.

\section{BIBLIOGRAPHIE}

Anscombre, J.-Cl. (1989) « Théorie de l'Argumentation, topoï, et structuration discursive », Revue Québécoise de Linguistique Vol. 18 n¹, 13-55.

Anscombre, J.-Cl. (1990) « Pourquoi un moulin à vent n'est pas un ventilateur », Langue française 86, 103-125.

Anscombre, J.-Cl. (2009) « Des adverbes d'énonciation aux marqueurs d'attitude énonciative : le cas de la construction tout + Adjectif », Langue française 161, 59-80.

Ducrot, O. \& al. (1980) Les mots du discours, Paris : Editions de minuit.

Gomez Jordana Ferary, S. (2011) «Décidément et decididamente : une évolution à deux vitesses? », Langages 184, 69-90.

Greebaum, S. (1969) Studies in English Adverbial Usage. Londres : Ed. Longman.

Guimier, Cl. (1996) Les adverbes du français. Le cas des adverbes en -ment, ParisGap :Ophrys.

Huang. S.-F. (1975) A Study of Adverbs. La Haye : Mouton.

Kleiber, G. (1994) Anaphores et pronoms, Louvain-la-Neuve : Duculot.

Melis, L. (1983) Les circonstants et la phrase, Louvain : Presses Universitaires de Louvain.

Molinier, Ch. (1990) «Une classification des adverbes en -ment », Langue française $88,28-40$.

Molinier, Ch. (2009) «Les adverbes d'énonciation : comment les définir et les sousclassifier », Langue française 161, 9-22.

Molinier, Ch. \& Levrier, F. (2000) Grammaire des adverbes. Description des formes en -ment. Genève-Paris : Droz.

Mørdrup, O. (1976) « Une analyse non-transformationnelle des adverbes en -ment », Revue Romane 11 : 317-333.

Rouanne, L. (2010) «Intensité et délocutivité dans les adverbes en -ment », Revue Romane 45, 45-69.

Schelling, M. (1982) «Quelques modalités de clôture : les conclusifs », Cahiers de linguistique française $4: 63-106$.

Schlyter, S. (1977) La place des adverbes en -ment en français, Thèse. Constance. 